\title{
CONSTITUINTES QUÍMICOS DE Ottonia corcovadensis MIQ. DA FLORESTA AMAZÔNICA - ATRIBUIÇÃO DOS DESLOCAMENTOS QUÍMICOS DOS ÁTOMOS DE HIDROGÊNIO E CARBONO
}

Valdir A. Facundo

Departamento de Química, Universidade Federal de Rondônia, Br 364, km 9,5, 78900-000 Porto Velho - RO

Selene M. Morais

Departamento de Química e Física, Centro de Ciências e Tecnologia, Universidade Estadual do Ceará,

Av. Paranjana 1700, 60740-000 Fortaleza - CE

Raimundo Braz Filho*

Setor de Química de Produtos Naturais, Laboratório de Ciências Químicas, Centro de Ciência e Tecnologia, Universidade Estadual do Norte Fluminense, Av. Alberto Lamego, 2000, 28013-600 Campos - RJ

CHEMICAL CONSTITUENTS OF Ottonia corcovadensis MIQ. FROM AMAZON FOREST $-{ }^{1} \mathrm{H}$ AND ${ }^{13} \mathrm{C}$ CHEMICAL SHIFT ASSIGNMENTS. In an ethanolic extract of leaves of Ottonia corcovadensis (Piperaceae) were identified sixteen terpenoids of essential oil and the three flavonoids 3',4',5,5',7-pentamethoxyflavone (1), 3', 4',5,7-tetramethoxyflavone (2) and 5-hydroxy3', 4',5',7-tetramethoxyflavone (3) and cafeic acid (4). Two amides (5 and $\mathbf{6})$ were isolated from an ethanolic extract of the roots. The structures were established by spectral analysis, meanly NMR (1D and 2D) and mass spectra. Extensive NMR analysis was also used to complete ${ }^{1} \mathrm{H}$ and ${ }^{13} \mathrm{C}$ chemical shift assignments of the flavonoids and amides. The components of the essential oil were identified by computer library search, retention indices and visual interpretation of mass spectra.

Keywords: Ottonia corcovadensis; Piperaceae; terpenoids; flavonoids; cafeic acid; amides.

\section{INTRODUÇÃO}

A espécie Ottonia corcovadensis Miq. (Piperaceae) é comumente encontrada no norte e nordeste do Brasil, sendo conhecida popularmente como João brandinho. No Estado do Acre, aparece como uma das principais espécies vegetais da medicina popular, onde as folhas são usadas para tratamento de reumatismo na forma de compressa e como chá para gripe e tosse ${ }^{1}$.

Este artigo descreve o estudo químico das folhas e das raízes de um espécime de Ottonia corcovadensis coletado em Porto Velho, Rondônia, Brasil. A análise do óleo essencial das folhas permitiu a identificação de dezesseis terpenóides (Tabela 1) através de cromatografia gasosa acoplada a espectrometria de massas (CG/EM). Os flavonóides 3',4',5,5',7-pentametoxiflavona (1), 3',4',5,7tetrametoxiflavona (2) e 5-diidroxi-3',4',5',7-tetrametoxiflavona (3) e o ácido cafeico (4) foram isolados do extrato etanólico das folhas, após a extração do óleo essencial por arraste de vapor. Do extrato etanólico das raízes foram isoladas as amidas piperovatina (5) e chingchengenamida (6). As estruturas dos flavonóides e das amidas foram estabelecidas com base na análise de dados espectrais, principalmente os obtidos de espectros de $\mathrm{RMN}{ }^{1} \mathrm{H}$ e ${ }^{13} \mathrm{C}(1 \mathrm{D} \text { e } 2 \mathrm{D})^{2}$ e de massas. A interpretação detalhada dos espectros $1 \mathrm{D}$ e 2D de RMN ${ }^{1} \mathrm{H}$ e ${ }^{13} \mathrm{C}$ foi também utilizada na atribuição inequívoca dos deslocamentos químicos dos três flavonóides (Tabelas 2 e 3) e das duas amidas (Tabela 4), além de permitir reconhecer a presença de todos sinais característicos da amida piperovatina (5) nos espectros da flavona 3 (componente predominante).

No sudeste do Brasil essa planta é conhecida como falso jaborandi, sendo suas raízes e ramos mastigados para aliviar dor de dente, devido à ação anestésica sobre a membrana mucosa da boca.
Investigação fitoquímica do extrato éter de petróleo das raízes de um espécime coletado na montanha do Corcovado, Rio de Janeiro, Brasil, registrou a presença de cinco amidas, piperovatina (5), piperlonguminina (7), corcovadina $(\mathbf{8})$, isopiperlonguminina $(\mathbf{9}) \mathrm{e}$ isocorcovadina (10). A atividade anestésica da planta foi atribuída à presença de piperovatina $(\mathbf{5})^{3}$.

A região onde foi coletado o material de Ottonia corcovadensis para nosso estudo encontra-se inserida em condição ambiental significativamente diferente da observada na capital do Estado do Rio de Janeiro, onde foram coletadas as raízes da mesma espécie para a investigação fitoquímica anterior ${ }^{3}$.<smiles>COc1cc(O)c2c(=O)cc(-c3cc(OC)c(OC)c(OC)c3)oc2c1</smiles><smiles>O=C(O)/C=C/c1ccc(O)c(O)c1</smiles><smiles></smiles><smiles>COc1ccc(CC=CC=CC(=O)NCC(C)C)cc1</smiles><smiles>CC(C)CNC(=O)C=CC=CCCc1ccc2c(c1)OCO2</smiles>
6<smiles>[R]OC(=O)OCC(C)(C)CNC(=O)C=CC=Cc1ccc2c(c1)OCO2</smiles>

*e-mail: braz@uenf.br

Figura 1. Substâncias naturais isoladas de Ottonia corcovadensis 


\section{RESULTADOS E DISCUSSÃO}

A análise do óleo essencial através de cromatografia gasosa acoplada a espectrômetro de massas (CG/EM), pesquisa computacional em biblioteca de substâncias Hewlett - Packard, índices de retenção e interpretação visual de espectros de massas ${ }^{4-6}$ possibilitou a identificação de dezesseis componentes (Tabela 1). Limoneno $(12,74 \%)$, trans-cariofileno $(13,63 \%)$, epibiciclosesquifilandreno $(10,25 \%)$ e germacreno B $(9,81 \%)$ destacaram-se como os componentes principais. Várias substâncias descritas como componentes dos óleos essenciais das espécies brasileiras Piper arboreum Aublet var. latifolium (CDC) e P. hispidum ${ }^{7}$ foram também encontrados em O. corcovadensis.

Os flavonóides 3',4',5,5',7-pentametoxiflavona (1) e 3',4',5,7tetrametoxiflavona (2), isolados como uma mistura, foram caracterizados principalmente com base nos espectros de $\mathrm{RMN}^{1} \mathrm{H}$ e ${ }^{13} \mathrm{C}$ (Tabela 2). $\mathrm{O}$ espectro de $\mathrm{RMN}^{1} \mathrm{H}$ mostrou os sinais dos átomos de hidrogênio do anel $\mathrm{B}$ de 1 em $\delta_{\mathrm{H}}$ 3,79 (s, MeO-3' e MeO-5'), 3,77 (s, MeO-4') e 6,86 (s, H-2'/H-6') e de 2 em $\delta_{\mathrm{H}} 3,80$ (s, MeO-3'), 3,78 (s, MeO-4'), 7,08 (d, J=2,2 Hz, H-2'), 6,75, (d, J=8,6 Hz, H-5') e 7,26, (dd, J=2,2 e 8,6 Hz, H-6'). As informações fornecidas pelos espectros de $\mathrm{RMN}^{13} \mathrm{C}\left(\left\{{ }^{1} \mathrm{H}\right\}\right.$ e DEPT135) e 2D de correlação heteronuclear (HMQC e HMBC) confirmaram estas deduções e permitiram a atribuição inequívoca dos deslocamentos químicos de todos os átomos de hidrogênio e carbono de cada uma das flavonas ( $\mathbf{1}$ e 2) presentes na mistura (Tabela 2). Com base nas intensidades relativas reveladas pela integração computacional dos sinais dos átomos
Tabela 1. Constituintes químicos do óleo essencial das folhas de $O$. corcovadensis

\begin{tabular}{lcc}
\hline Constituintes & IR* & Percentagem \\
\hline$\beta$-pineno & 957 & 6,4 \\
Felandreno & 986 & 7,8 \\
$p$-cimeno & 1006 & 3,8 \\
Limoneno & 1012 & 12,7 \\
$\alpha$-cubebeno & 1339 & 1,8 \\
$\alpha$-copaeno & 1364 & 2,5 \\
$\beta$-elemeno & 1379 & 1,9 \\
trans-cariofileno & 1406 & 13,6 \\
(Z)- $\beta$-farneseno & 1438 & 5,6 \\
Aloaromadendreno & 1443 & 1,2 \\
epi-biciclosesquifelandreno & 1462 & 10,3 \\
Biciclogermacreno & 1475 & 7,9 \\
$\beta$-bisaboleno & 1484 & 1,3 \\
$\gamma$-cadineno & 1490 & 2,0 \\
$\delta$-cadineno & 1498 & 2,3 \\
germacreno B & 1528 & 9,8 \\
\hline Total identificado & & 90,9
\end{tabular}

Total identificado

90,9

*IR=Índices de retenção. Os constituintes estão descritos em ordem crescente de eluição em coluna não polar.

Tabela 2. Dados de RMN ${ }^{1} \mathrm{H}$ e ${ }^{13} \mathrm{C}$, inclusive de correlação heteronuclear ${ }^{1} \mathrm{H}^{-13} \mathrm{C}-\mathrm{COSY}-{ }^{\mathrm{n}} \mathrm{J}_{\mathrm{CH}}(\mathrm{n}=1$, HMQC; $\mathrm{n}=2$ e 3 , HMBC), de $\mathbf{1}$ e 2 em $\mathrm{CDCl}_{3}$ e TMS como referência interna. Deslocamentos químicos em $\delta$ (ppm) e constantes de acoplamento (J, entre parênteses) em Hz*

\begin{tabular}{|c|c|c|c|c|c|c|c|c|}
\hline & \multicolumn{4}{|c|}{1} & \multicolumn{4}{|c|}{2} \\
\hline & $\delta_{\mathrm{C}}$ & $\delta_{\mathrm{H}}$ & ${ }^{2} \mathrm{~J}_{\mathrm{CH}}$ & ${ }^{3} \mathbf{J}_{\mathrm{CH}}$ & $\delta_{\mathrm{C}}$ & $\delta_{\mathrm{H}}$ & ${ }^{2} \mathbf{J}_{\mathrm{CH}}$ & ${ }^{3} \mathrm{~J}_{\mathrm{CH}}$ \\
\hline \multicolumn{9}{|l|}{$\mathrm{C}$} \\
\hline 2 & 160,25 & - & $\mathrm{H}-3$ & H-2', H-6' & 160,42 & - & $\mathrm{H}-3$ & $2 \mathrm{H}-2^{\prime}, 6^{\prime}$ \\
\hline 4 & 177,45 & - & $\mathrm{H}-3$ & - & 177,40 & - & $\mathrm{H}-3$ & - \\
\hline 5 & 160,64 & - & H-6 & $\mathrm{MeO}-5$ & 160,61 & - & H-6 & $\mathrm{MeO}-5$ \\
\hline 7 & 164,97 & - & H-6, H-8 & MeO-7 & 164,83 & - & H-6, H-8 & MeO-7 \\
\hline 9 & 159,64 & - & H-8 & - & 159,61 & - & H-8 & - \\
\hline 10 & 108,91 & - & - & H-3, H-6, H-8 & 108,91 & - & - & H-3, H-6, H-8 \\
\hline 1, & 126,49 & - & H-2'/H- 6' & $\mathrm{H}-3$ & 123,68 & - & $\mathrm{H}-2$ & H-3, H-5 \\
\hline $3^{\prime}$ & 153,35 & - & H-2' & MeO-3' & 149,02 & - & $\mathrm{H}-2$ & H-5', MeO-3' \\
\hline $4^{\prime}$ & 140,64 & - & - & 2H-2',6; MeO-4' & 151,57 & - & $\mathrm{H}-5$ & 2H-2',6; MeO-4' \\
\hline $5^{\prime}$ & 153,35 & - & H-6' & $\mathrm{MeO}-5$ & - & - & - & - \\
\hline \multicolumn{9}{|l|}{ CH } \\
\hline 3 & 108,49 & $6,41(\mathrm{~s})$ & - & - & 107,54 & $6,38(\mathrm{~s})$ & - & - \\
\hline 6 & 96,06 & $6,16(\mathrm{~d}, 2,2)$ & & $\mathrm{H}-8$ & 95,96 & $6,15(\mathrm{~d}, 2,2)$ & - & $\mathrm{H}-8$ \\
\hline 8 & 92,81 & $6,36(\mathrm{~d}, 2,2)$ & & H-6 & 92,76 & $6,34(\mathrm{~d}, 2,2)$ & - & H-6 \\
\hline $2^{\prime}$ & 103,10 & $6,86(\mathrm{~s})$ & & - & 108,26 & $7,08(\mathrm{~d}, 2,0)$ & - & H-6' \\
\hline $5^{\prime}$ & - & - & & - & 110,92 & $6,75(\mathrm{~d}, 8,6)$ & - & - \\
\hline 6 & 103,10 & $6,86(\mathrm{~s})$ & & - & 119,33 & $7,26(\mathrm{dd}, 8,6$ e 2,0$)$ & - & $\mathrm{H}-2$ \\
\hline $\mathrm{MeO}$ & & & & - & & & & \\
\hline 5 & 55,96 & $3,77(\mathrm{~s})$ & & - & 55,96 & $3,77(\mathrm{~s})$ & - & - \\
\hline 7 & 55,78 & $3,75(\mathrm{~s})$ & & - & 55,73 & $3,74(\mathrm{~s})$ & - & - \\
\hline $3^{\prime}$ & 56,24 & $3,79(\mathrm{~s})$ & & - & 55,73 & $3,80(\mathrm{~s})$ & - & - \\
\hline $4^{\prime}$ & 60,92 & $3,77(\mathrm{~s})$ & & - & 56,24 & $3,78(\mathrm{~s})$ & - & - \\
\hline $5^{\prime}$ & 56,24 & $3,79(\mathrm{~s})$ & & - & - & - & - & - \\
\hline
\end{tabular}

*Análise comparativa dos espectros de $\mathrm{RMN}^{13} \mathrm{C}-\mathrm{HBBD}$ e $\mathrm{RMN}{ }^{13} \mathrm{C}$-DEPT foi usada para reconhecer os átomos de carbono quaternários, metínicos e metoxílicos. Deslocamentos químicos e constantes de acoplamento (J) dos átomos de hidrogênio foram deduzidos dos espectros $1 \mathrm{D}$ de $\mathrm{RMN}^{1} \mathrm{H}$. 
Tabela 3. Dados de $\mathrm{RMN}{ }^{1} \mathrm{H} \mathrm{e}{ }^{13} \mathrm{C}$ da 5-hidroxi-7,3', 4',5' -tetrametoxiflavona (3) comparados com os valores de deslocamentos químicos dos átomos da 5,7-diidroxi-3', $4^{\prime}, 5^{\prime}$-trimetoxiflavona ${ }^{8}, \mathrm{em}^{-} \mathrm{CDCl}_{3}^{*}$

\begin{tabular}{|c|c|c|c|c|c|}
\hline & \multicolumn{4}{|c|}{3} & \multirow{2}{*}{$\begin{array}{c}\text { 5,7-diidroxi-3', } 4^{\prime}, 5^{\prime}- \\
\text { trimetoxiflavona } \\
\delta_{\mathrm{C}}\end{array}$} \\
\hline & $\delta_{\mathrm{C}}$ & $\delta_{\mathrm{H}}$ & ${ }^{2} \mathbf{J}_{\mathrm{CH}}$ & ${ }^{3} \mathbf{J}_{\mathrm{CH}}$ & \\
\hline \multicolumn{6}{|l|}{$\mathbf{C}$} \\
\hline 2 & 163,88 & - & $\mathrm{H}-3$ & H-2'/H-6' & 164,2 \\
\hline 4 & 182,36 & - & H-3 & & 181,7 \\
\hline 5 & 162,26 & - & HO-5; H-6 & & 161,4 \\
\hline 7 & 165,67 & - & H-6; H-8 & $\mathrm{MeO}-7$ & 163,0 \\
\hline 9 & 157,76 & - & H-8 & & 157,4 \\
\hline 10 & 105,69 & - & & H-3; HO-5; H-6; H-8 & 104,8 \\
\hline 1 ' & 126,57 & - & H-2'/H-6' & $\mathrm{H}-3$ & 125,9 \\
\hline 3, & 153,67 & - & H-2’ & $\mathrm{MeO}-3$ & 153,2 \\
\hline $4{ }^{\prime}$ & 141,47 & - & & H-2'/H-6'; MeO-4' & 141,4 \\
\hline 5 & 153,67 & - & H-6' & $\mathrm{MeO}-5$ & 153,2 \\
\hline \multicolumn{6}{|l|}{ CH } \\
\hline 3 & 105,69 & $6,58(\mathrm{~s})$ & & & 103,9 \\
\hline 6 & 98,26 & $6,36(\mathrm{~d}, \mathrm{~J}=2,4)$ & & $\mathrm{HO}-5 ; \mathrm{H}-8$ & 99,0 \\
\hline 8 & 92,79 & $6,48(\mathrm{~d}, \mathrm{~J}=2,4)$ & & H-6 & 94,2 \\
\hline $2^{\prime}, 6^{\prime}$ & 103,82 & $7,06(\mathrm{~s})$ & & & 104,8 \\
\hline \multicolumn{6}{|l|}{$\mathrm{MeO}$} \\
\hline 7 & 55,90 & $3,87(\mathrm{~s})$ & & & \\
\hline 3 ' & 56,43 & $3,99(\mathrm{~s})$ & & & \\
\hline $4{ }^{\prime}$ & 61,11 & $3,91(\mathrm{~s})$ & & & \\
\hline 5 & 56,43 & 3,99 (s) & & & \\
\hline $\mathrm{HO}-5$ & - & $12,07(\mathrm{~s})$ & & & \\
\hline
\end{tabular}

*Análise comparativa dos espectros de $\mathrm{RMN}^{13} \mathrm{C}-\mathrm{HBBD}$ e $\mathrm{RMN}{ }^{13} \mathrm{C}$-DEPT foi usada para reconhecer os átomos de carbono quaternários, metínicos, metilênicos e metilicos. Deslocamentos químicos e constantes de acoplamento (J) dos átomos de hidrogênio foram deduzidos dos espectros $1 \mathrm{D}$ de $\mathrm{RMN}^{1} \mathrm{H}$.

de hidrogênio H-2'/H-6' $\left(\delta_{\mathrm{H}} 6,86\right)$ de 1 e H-5' $\left(\delta_{\mathrm{H}} 6,75\right)$ de 2 no espectro 1D de $\mathrm{RMN}^{1} \mathrm{H}$ da mistura calculou-se as percentagens aproximadas das duas flavonas: $60,2 \%$ (1) e $39,8 \%$ (2).

A estrutura 5-hidroxi-3',4',5',7-tetrametoxiflavona (3) foi definida com base na análise dos espectros $1 \mathrm{D}$ e $2 \mathrm{D}$ de $\mathrm{RMN}{ }^{1} \mathrm{H}$ e ${ }^{13} \mathrm{C}$, envolvendo a comparação com dados de 2 (Tabela 2) e de $\mathrm{RMN}^{13} \mathrm{C}$ da 5,7-diidroxi-3',4',5'-trimetoxiflavona ${ }^{8}$ (Tabela 3). A atribuição inequívoca dos deslocamentos químicos dos átomos de hidrogênio e carbono de 3 baseou-se também em correlações a longa distância de carbono e hidrogênio $\left({ }^{2} \mathrm{~J}_{\mathrm{CH}} \mathrm{e}^{3} \mathrm{~J}_{\mathrm{CH}}\right)$ observadas no espectro 2D HMBC (Tabela 3). A comparação dos dados de $\mathrm{RMN}^{13} \mathrm{C}$ de 3 e 5,7-diidroxi3',4',5'-trimetoxiflavona revelou efeito $\gamma$ esperado do grupo metoxílico MeO-7 nos carbonos CH-6 e CH-8 (3), localizados em posição orto (Tabela 3). Após a definição estrutural da flavona 3 , foi possível verificar a presença de todos os sinais característicos da amida piperovatina (5) nos $1 \mathrm{D}$ e $2 \mathrm{D}$ de $\mathrm{RMN}^{1} \mathrm{H} \mathrm{e}^{13} \mathrm{C}$. O ácido cafeico (4) foi identificado através dos dados fornecidos pelos espectros de $\mathrm{RMN}^{1} \mathrm{H}$ e ${ }^{13} \mathrm{C}$ comparados com valores descritos na literatura9.

As flavonas 1, 2 e 3 não foram encontradas em outras espécies dos gêneros Ottonia e Piper. O ácido cafeico (4) foi isolado de Piper nigrum. A espécie $O$. corcovadensis revela estreita relação botânica com espécies do gênero Piper. O número de flavonas isoladas de Piper pode ser classificado como relativamente reduzido, destacando-se flavonas tri- e tetraoxigenadas ${ }^{10}$. A 3', 4',5,5,7-pentametoxiflavona (1) e a 3',4',5,7-tetrametoxiflavona (2) foram isoladas das espécies Merrillia caloxylosa (família Rutaceae), Murraya paniculata (família Rutaceae) e Bauhinia championii (família Leguminosae), e 5-hidroxi-3',4',5',7-tetrametoxiflavona (3) de Webera corymbosa ${ }^{11}$.

As amidas 5 e 6, isoladas como uma mistura do extrato etanólico das raízes, foram caracterizadas através dos espectros de $\mathrm{RMN}^{1} \mathrm{H}$ (1D e $\left.2 \mathrm{D}{ }^{1} \mathrm{H}-{ }^{1} \mathrm{H}-\mathrm{COSY}\right), \mathrm{RMN}{ }^{13} \mathrm{C}\left(\left\{{ }^{1} \mathrm{H}\right\}\right.$ e DEPT135), HMQC e HMBC e cromatografia gasosa acoplada a espectrômetro de massas. As amidas piperovatina (5) e chingchengenamida (6) revelaram, respectivamente, $\mathrm{Tr}=11,676$ e 12,742 $\mathrm{min}$ no cromatograma e picos correspondentes a íons moleculares em $\mathrm{m} / \mathrm{z}, 273(27,9 \%)$ e $301(9,3 \%)$ nos espectros de massas (Figura 2). O pico base em $\mathrm{m} / \mathrm{z}, 173$ obser-<smiles>COc1ccc(CC=CC=CC=CC(=O)NCC(C)C)cc1</smiles><smiles>C=CC=CCc1ccc(OC)c(C=CC=C)c1</smiles>

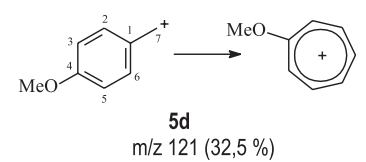<smiles>CC(C)CNC(=O)/C=C/C=C/CCc1ccc2c(c1)OCO2</smiles><smiles>[13CH2]c1ccc2c(c1)OCO2</smiles>

Figura 2. Fragmentos atribuídos aos picos principais observados nos espectros de massas das amidas naturais 5 e 6 (Figura 1) 
vado no espectro de massas de $\mathbf{5}$ foi atribuído ao fragmento $\mathbf{5 c}$, sendo a localização do grupo metoxila baseada também em argumentação biogenética. $\mathrm{O}$ espectro de massas de $\mathbf{6}$ revelou o pico base em $\mathrm{m} / z 135(\mathbf{6 b})$. A análise comparativa dos espectros de $\mathrm{RMN}^{13} \mathrm{C}\left(\left\{{ }^{1} \mathrm{H}\right\}\right.$ e DEPT135) permitiu identificar os sinais de átomos de carbono quaternários, metínicos, metilênicos e metílicos para cada uma das substâncias presentes na mistura (Tabela 4). O espectro de $\mathrm{RMN}^{1} \mathrm{H}$ mostrou sinais de grupo isobutila em $\delta_{\mathrm{H}} 0,92$ (d, J=6,7 Hz, 3H-3' e $3 \mathrm{H}-4^{\prime}$ ), 1,79 (m, H-2') e 3,15 (t, 6,5 Hz, 2H-1'), revelando intensidades relativas compatíveis com a presença deste grupo nas moléculas dos dois componentes. Os espectros $1 \mathrm{D}$ de $\mathrm{RMN}^{1} \mathrm{He}^{13} \mathrm{C}$ revelaram como diferença significativa entre as duas substâncias a presença de um anel aromático para-dissubstituído (para-metoxifenila) em 5 [sistema AA'BB' no espectro de $\mathrm{RMN}^{1} \mathrm{H}$ : $\delta_{\mathrm{H}} 7,07$ (d=8,3 Hz, H-2/ H-6) e 6,84 (d=8,3 Hz, H-3/H-5), além do singleto em $\delta_{\mathrm{H}} 3,90$ correspondente aos três hidrogênios do grupo metoxila; $\delta_{\mathrm{C}} 129,81(\mathrm{CH}-$ 2/CH-6), 114,20 (CH-3/CH-5) e 55,49 (MeO-4) no espectro de $\mathrm{RMN}^{13} \mathrm{C}$ ] e um metilenodioxifenila envolvido num sistema $1,3,4-$ trissubstituído de 6 [sistema $\mathrm{ABX}$ no espectro de $\mathrm{RMN}^{1} \mathrm{H}: \delta_{\mathrm{H}} 6,62$ (sl, H-2), 6,84 (d=7,8 Hz, H-5) e 6,60 (dl, d=7,8 Hz, H-6), além do singleto em $\delta_{\mathrm{H}} 5,92$ correspondente aos dois hidrogênios do grupo metilenodioxílico; $\delta_{\mathrm{C}}$ 109,02 (CH-2), 108,96 (CH-5), 121,38 (CH6) e $100,99\left(\mathrm{OCH}_{2} \mathrm{O}\right)$ no espectro de $\left.\mathrm{RMN}^{13} \mathrm{C}\right]$, em acordo com os fragmentos $\mathbf{5 b}, \mathbf{5 c}, \mathbf{5 d}$ e $\mathbf{6 b}$ deduzidos da análise dos espectros de massas. A diferença adicional entre as amidas 5 e $\mathbf{6}$ foi justificada com relativa facilidade pela presença de mais um grupo metilênico $\left(\mathrm{CH}_{2}-7 \mathrm{a}\right)$ na cadeia carbônica de $6\left([\mathrm{M}]^{++} 301\right)$, confirmada pelo espectro HMQC através da correlação heteronuclear indicando a interação do sinal em $\delta_{\mathrm{C}} 35,16\left(\mathrm{CH}_{2}-7\right.$ e $\left.\mathrm{CH}_{2}-7 \mathrm{a}\right)$ com os sinais em $\delta_{\mathrm{H}} 2,66(\mathrm{t}, 2 \mathrm{H}-7)$ e 2,42 (q, 2H-7a). O carbono metilênico $\mathrm{CH}_{2}-7\left(\delta_{\mathrm{C}}\right.$ $38,48)$ de 5 apareceu claramente correlacionado com o sinal em $\delta_{\mathrm{H}}$ 3,42 (d, J=6,1 Hz, 2H-7). O espectro $2 \mathrm{D}{ }^{1} \mathrm{H}-{ }^{1} \mathrm{H}-\mathrm{COSY}$ revelou com clareza as interações spin-spin dos átomos de hidrogênio $2 \mathrm{H}-7 \mathrm{a}\left(\delta_{\mathrm{H}}\right.$ $2,42, \mathrm{t}, \mathrm{J}=7,4 \mathrm{~Hz})$ com os $2 \mathrm{H}-7\left(\delta_{\mathrm{H}} 2,66, \mathrm{q}, \mathrm{J}=7,4 \mathrm{~Hz}\right)$ e o $\mathrm{H}-8\left(\delta_{\mathrm{H}}\right.$ $6,17, \mathrm{~s})$ da amida $\mathbf{6}$. O maior $\delta_{\mathrm{H}}$ dos hidrogênios $2 \mathrm{H}-7\left(\delta_{\mathrm{H}} 3,42\right)$ de 5 pode ser justificado pela sua localização em posição recebendo a influência dos efeitos anisotrópicos do grupo arila e da ligação dupla (diretamente ligados ao $\left.\mathrm{CH}_{2}-7\right)$, enquanto os $2 \mathrm{H}-7\left(\delta_{\mathrm{H}} 2,66\right)$ sentem efeito anisotrópico do grupo arila (maior efeito anisotrópico) e $2 \mathrm{H}$ $7 \mathrm{a}\left(\delta_{\mathrm{H}} 2,42\right)$ de 6 da ligação dupla (menor efeito anisotrópico). Outras correlações diretas de carbono e hidrogênio $\left({ }^{1} \mathrm{~J}_{\mathrm{CH}}\right)$ observadas no espectro HMQC e a longa distância $\left({ }^{2} \mathrm{~J}_{\mathrm{CH}} \mathrm{e}^{3} \mathrm{~J}_{\mathrm{CH}}\right)$ no HMBC encontram-se resumidas na Tabela 4, contendo a completa atribuição dos deslocamentos dos átomos de carbono e hidrogênio de cada uma das duas amidas (5 e 6). Com base na intensidade relativa do sinal dos átomos de hidrogênio do grupo $\mathrm{OCH}_{2} \mathrm{O}\left(\delta_{\mathrm{H}} 5,92\right)$ de $\mathbf{6}$ e do H-2/H-6 $\left(\delta_{\mathrm{H}} 7,07\right)$ de $\mathbf{5}$ foi possível calcular as percentagens aproximadas das duas isobutilamidas presentes na mistura: $54,7 \%$ de $\mathbf{6}$ e $45,3 \%$ de $\mathbf{5}$.

Tabela 4. Dados de $\mathrm{RMN}^{1} \mathrm{H}(400 \mathrm{MHz}) \mathrm{e}^{13} \mathrm{C}(100 \mathrm{MHz})$ das amidas 5 e 6, incluindo resultados obtidos de espectros de correlação heteronuclear ${ }^{1} \mathrm{H}_{-}{ }^{13} \mathrm{C}-\mathrm{COSY}-\mathrm{J}_{\mathrm{CH}}(\mathrm{n}=1, \mathrm{HMQC} ; \mathrm{n}=2$ e $3, \mathrm{HMBC})$, em $\mathrm{CDCl}_{3}$ e sinais residuais do solvente usados como referências internas $\left(\delta_{\mathrm{H}} 7,26\right.$ e $\delta_{\mathrm{C}}$ $70,00)$. Deslocamentos químicos em $\delta$ (ppm) e constantes de acoplamento ( $J$, entre parênteses) em $\mathrm{Hz}^{*}$

\begin{tabular}{|c|c|c|c|c|c|c|c|c|}
\hline & \multicolumn{4}{|c|}{6} & \multicolumn{4}{|c|}{5} \\
\hline & \multicolumn{2}{|c|}{ HMQC } & \multicolumn{2}{|c|}{ HMBC } & \multicolumn{2}{|c|}{ HMQC } & \multicolumn{2}{|c|}{ HMBC } \\
\hline & $\delta_{\mathrm{C}}$ & $\delta_{\mathrm{H}}$ & ${ }^{2} \mathbf{J}_{\mathrm{CH}}$ & ${ }^{3} \mathbf{J}_{\mathrm{CH}}$ & $\delta_{\mathrm{C}}$ & $\delta_{\mathrm{H}}$ & ${ }^{2} \mathbf{J}_{\mathrm{CH}}$ & ${ }^{3} \mathrm{~J}_{\mathrm{CH}}$ \\
\hline \multicolumn{9}{|l|}{$\mathrm{C}$} \\
\hline 1 & 135,29 & - & $2 \mathrm{H}-7$ & $\mathrm{H}-5 ; 2 \mathrm{H}-7 \mathrm{a}$ & 131,32 & - & & H-3/H-5; H-8 \\
\hline 3 & 147,79 & - & $\mathrm{H}-2$ & $\mathrm{H}-5 ; \mathrm{OCH}_{2} \mathrm{O}$ & - & - & - & - \\
\hline 4 & 145,94 & - & $\mathrm{H}-5$ & $\mathrm{H}-2 ; \mathrm{H}-6 ; \mathrm{OCH}_{2} \mathrm{O}$ & 158,39 & - & $\mathrm{H}-3 / \mathrm{H}-5$ & $\mathrm{H}-2 / \mathrm{H}-6 ; \mathrm{MeO}-4$ \\
\hline 12 & 166,61 & - & $\mathrm{H}-11$ & $2 \mathrm{H}-1^{\prime} ; \mathrm{H}-10$ & 166,53 & - & H-11 & 2H-1'; H-10 \\
\hline \multicolumn{9}{|l|}{$\mathrm{CH}$} \\
\hline 2 & 109,02 & $6,62(\mathrm{sl})$ & & $\mathrm{H}-6 ; 2 \mathrm{H}-7$ & 129,81 & $7,07(\mathrm{~d}, 8,3)$ & & $2 \mathrm{H}-7$ \\
\hline 3 & - & - & - & - & 114,20 & $6,84(\mathrm{~d}, 8,3)$ & & \\
\hline 5 & 108,96 & $6,84(\mathrm{~d}, 7,8)$ & & & 114,20 & $6,84(\mathrm{~d}, 8,3)$ & & \\
\hline 6 & 121,38 & $6,60(\mathrm{dl}, 7,8)$ & & $\mathrm{H}-2 ; 2 \mathrm{H}-7$ & 129,81 & $7,07(\mathrm{~d}, 8,3)$ & & $2 \mathrm{H}-7$ \\
\hline 8 & 141,70 & $6,06(\mathrm{td}, 15,0,7,4)$ & $2 \mathrm{H}-7 \mathrm{a}$ & $2 \mathrm{H}-7 ; \mathrm{H}-10$ & 140,96 & $6,17(\mathrm{td}, 15,0,6,1)$ & $2 \mathrm{H}-7$ & H-10 \\
\hline 9 & 129,10 & 6,14 & H-8; H-10 & $2 \mathrm{H}-7 \mathrm{a} ; \mathrm{H}-11$ & 129,27 & 6,11 & $\mathrm{H}-10$ & $2 \mathrm{H}-7 ; \mathrm{H}-11$ \\
\hline 10 & 141,35 & $7,17(\mathrm{dd}, 15,0,10,5)$ & H-9 & H-8 & 141,19 & $7,20(\mathrm{dd}, 15,0,10,2)$ & & H-8 \\
\hline 11 & 122,51 & $5,77(\mathrm{~d}, 15,0)$ & $\mathrm{H}-10$ & $\mathrm{H}-9$ & 122,92 & $5,80(\mathrm{~d})$ & H-10 & $\mathrm{H}-9$ \\
\hline $2^{\prime}$ & 28,82 & $1,79(\mathrm{~m})$ & $2 \mathrm{H}-1^{\prime} ; 3 \mathrm{H}-3^{\prime} ; 3 \mathrm{H}-4$ & & 28,82 & $1,79(\mathrm{~m})$ & $2 \mathrm{H}-1^{\prime} ; 3 \mathrm{H}-3^{\prime} ; 3 \mathrm{H}-4$ ' & \\
\hline \multicolumn{9}{|l|}{$\mathrm{CH}_{2}$} \\
\hline $\mathrm{OCH}_{2} \mathrm{O}$ & 100,99 & $5,92(\mathrm{~s})$ & & & - & - & - & - \\
\hline $7^{2}$ & 35,16 & $2,66(t, 7,4)$ & & H-2; H-6 & 38,48 & $3,42(\mathrm{~d}, 6,1)$ & $\mathrm{H}-8$ & H-2/H-6; H-9 \\
\hline $7 \mathrm{a}$ & 35,16 & $2,42(\mathrm{q}, 7,4)$ & $\mathrm{H}-8$ & H-9 & - & - & - & - \\
\hline 1 ' & 47,18 & $3,15(\mathrm{t}, 6,7)$ & $\mathrm{H}-2^{\prime}$ & $3 \mathrm{H}-3^{\prime} ; 3 \mathrm{H}-4^{\prime}$ & 47,18 & $3,15(\mathrm{t}, 6,7)$ & $\mathrm{H}-2^{\prime}$ & $3 \mathrm{H}-3^{\prime} ; 3 \mathrm{H}-4^{\prime}$ \\
\hline \multicolumn{9}{|l|}{$\mathrm{CH}_{3}$} \\
\hline $\mathrm{MeO}-4$ & - & - & - & - & 55,49 & $3,90(\mathrm{~s})$ & & \\
\hline $3^{\prime}, 4^{\prime}$ & 20,33 & $0,92(d, 6,7)$ & $\mathrm{H}-2$, & $2 \mathrm{H}-1$ & 20,33 & $0,92(\mathrm{~d}, 6,7)$ & $\mathrm{H}-2$ & $2 \mathrm{H}-1$ \\
\hline
\end{tabular}

*Análise comparativa dos espectros de $\mathrm{RMN}{ }^{13} \mathrm{C}-\mathrm{HBBD}$ e $\mathrm{RMN}{ }^{13} \mathrm{C}$-DEPT foi usada para reconhecer os átomos de carbono quaternários, metínicos, metilênicos e metílicos. Deslocamentos químicos e constantes de acoplamento (J) dos átomos de hidrogênio foram deduzidos dos espectros $1 \mathrm{D}$ de $\mathrm{RMN}^{1} \mathrm{H}$. sl=singleto largo. Sinais de átomos de hidrogênio sem indicação de multiplicidade indicam sobreposição e deslocamentos químicos deduzidos dos espectros $2 \mathrm{D}{ }^{1} \mathrm{H}-{ }^{1} \mathrm{H}-\mathrm{COSY}$ e ${ }^{1} \mathrm{H}^{-13} \mathrm{C}-\mathrm{COSY}{ }^{-} \mathrm{J}_{\mathrm{CH}} \cdot$ 
Após a atribuição inequívoca dos $\delta_{\mathrm{C}}$ e $\delta_{\mathrm{H}}$ de $\mathbf{5}$ e $\mathbf{6}$, verificou-se que a piperovatina $(\mathbf{5})$ e a chinchengenamida (6) foram isoladas recentemente também de Piper piscatoru e P. falconare (família Piperaceae $)^{12,13}$, tornando-se possível a análise comparativa dos dados de $\mathrm{RMN}^{1} \mathrm{H}(400 \mathrm{MHz})$ e ${ }^{13} \mathrm{C}(100 \mathrm{MHz})$ com os valores descritos na literatura para as duas amidas $\left(\mathrm{CDCl}_{3}\right)$. As pequenas diferenças verificadas classificam-se na faixa do erro experimental, considerando-se também a obtenção dos nossos espectros em aparelho de mais alta resolução $\left[500\left({ }^{1} \mathrm{H}\right)\right.$ e $\left.125\left({ }^{13} \mathrm{C}\right) \mathrm{MHz}\right]$. Os dados descritos na Tabela 4 servem também para confirmação dos valores da literatura $^{12,13}$, já que a nossa análise inclui resultados obtidos de experiências bidimensionais (2D) de correlação homonuclear $\left({ }^{1} \mathrm{H}-{ }^{1} \mathrm{H}-\mathrm{COSY}\right)$ e heteronuclear $\left[{ }^{1} \mathrm{H}^{-13} \mathrm{C}^{-\mathrm{COSY}}{ }^{\mathrm{n}} \mathrm{J}_{\mathrm{CH}}(\mathrm{n}=1, \mathrm{HMQC}\right.$; $\mathrm{n}=2$ e $\left.3, \mathrm{HMBC})\right]$.

\section{PARTE EXPERIMENTAL}

\section{Procedimentos experimentais gerais}

Os espectros de RMN foram obtidos nos aparelhos Bruker Avance $500\left({ }^{1} \mathrm{H}: 500 \mathrm{MHz} ;{ }^{13} \mathrm{C}: 125 \mathrm{MHz}\right)$ e Jeol - $400\left({ }^{1} \mathrm{H}: 400\right.$ $\mathrm{MHz} ;{ }^{13} \mathrm{C}$ : $\left.100 \mathrm{MHz}\right)$. Os espectros $1 \mathrm{D}$ de $\mathrm{RMN}^{1} \mathrm{H}$ e $\mathrm{RMN}^{13} \mathrm{C}$ foram obtidos em condições padronizadas (detecção direta DUAL- ${ }^{1} \mathrm{H} /{ }^{13} \mathrm{C}$ $5 \mathrm{~mm}$ ); as experiências 2D foram acumuladas e processadas em um computador Aspectx32 com sofware da Bruker; seqüências padronizadas de pulso foram usadas para os espectros ${ }^{1} \mathrm{H}-{ }^{1} \mathrm{H}-\mathrm{COSY}$ (PO $45^{\circ}, \mathrm{SW} 8000 \mathrm{~Hz}, 1 \mathrm{~K}$ x 128 aquisições e $1 \mathrm{~K}$ x 512 no processamento); para a experiência homonuclear $2 \mathrm{D}{ }^{1} \mathrm{H}-{ }^{1} \mathrm{H}-\mathrm{NOESY}$ o programa NOESYTP (tempo de mixing $650 \mathrm{~ms}$ e intervalo de relaxamento $3 \mathrm{~s}$ ) foi utilizado após a solução ser borbulhada com $\mathrm{N}_{2}$; espectros de correlação heteronuclear 2D ${ }^{1} \mathrm{H}-{ }^{13} \mathrm{C}-\mathrm{HMQC}-{ }^{1} \mathrm{~J}_{\mathrm{CH}}$ e ${ }^{12} \mathrm{H}^{-13} \mathrm{C}-\mathrm{HMBC}-$ ${ }^{\mathrm{n}} \mathrm{J}_{\mathrm{CH}}(\mathrm{n}=2 \mathrm{e} 3)$ foram obtidos com o programa $\operatorname{INVBTP}\left({ }^{1} \mathrm{~J}_{\mathrm{CH}}=145 \mathrm{~Hz}\right.$, $f_{2} 30118 \mathrm{~Hz}$ e $f_{1} 8012 \mathrm{~Hz}$, intervalo de relaxamento $2 \mathrm{~s}$ ) e (MJ $52 \mathrm{~ms}$, ${ }^{\mathrm{n}} \mathrm{CH}_{\mathrm{CH}}=7 \mathrm{~Hz}, f_{2} 3020 \mathrm{~Hz}$ e $f_{1} 8012 \mathrm{~Hz}$, intervalo de relaxamento $2 \mathrm{~s}$ ). Os espectros de massas foram registrados por impacto eletrônico $(70 \mathrm{eV})$ em um aparelho GC/MS Hewlett - Packard 5971 usando coluna capilar ( $30 \mathrm{~m}$ x 0,25 mm) dimetilpolisiloxano BD-1, He como gás de arraste e as temperaturas de $250{ }^{\circ} \mathrm{C}$ no injetor, $200{ }^{\circ} \mathrm{C}$ no detector e variação de $1 \%$ min entre $35-180{ }^{\circ} \mathrm{C}$ e $10{ }^{\circ} \mathrm{C} /$ min entre 180 $-250{ }^{\circ} \mathrm{C}$ na coluna. Nas separações cromatográficas em coluna usouse sílica gel da Aldrich ou Merck com granulação adequada. As placas cromatográficas foram reveladas com luz UV ( $\left.\lambda_{\max } 254 \mathrm{~nm}\right)$, vapores de iodo e/ou solução alcoólica de vanilina e ácido sulfúrico.

\section{Planta}

O material vegetal foi coletado no sítio dos nove vegetais em Porto Velho, Rondônia, Brasil. A identificação botânica foi realizada no Jardim Botânico do Rio de Janeiro. Uma exsicata da espécie encontra-se depositada no herbário da Universidade Federal de Rondônia, Rondônia, Brasil, sob o número 032.

\section{Extração e separação}

O óleo essencial das folhas (500 g) de O. corcovadensis foi obtido por arraste de vapor d'água, fornecendo um óleo incolor com rendimento de $1,2 \%$. As folhas (500 g) usadas para extração do óleo essencial foram submetidas a secagem numa temperatura entre $60 \mathrm{e}$
$70{ }^{\circ} \mathrm{C}$ e extraídas com etanol na temperatura ambiente durante três dias. Destilação do etanol sob vácuo forneceu $26 \mathrm{~g}$ de material. Este material foi misturado com sílica gel (100 g), colocado em coluna cromatográfica e eluído sob pressão reduzida com hexano, clorofórmio, acetato de etila e metanol. A fração clorofórmica foi tratada com uma mistura de $\mathrm{MeOH}$ e $\mathrm{H}_{2} \mathrm{O}$ (90:10) e mantida num refrigerador durante uma noite para eliminar principalmente a clorofila. $\mathrm{O}$ material praticamente livre de clorofila foi seco e cromatografado em coluna de sílica gel usando como eluentes hexano e clorofórmio em misturas de polaridade crescente, obtendo-se uma mistura dos flavonóides $\mathbf{1}+\mathbf{2}$ e $\mathbf{3}$. O ácido cafeico (4) foi isolado do extrato acetato de etila através de cromatografia em coluna de sílica gel usando como eluente hexano e clorofórmio em misturas de polaridade crescente.

As raízes secas e moídas ( $650 \mathrm{~g}$ ) foram extraídas com etanol a temperatura ambiente durante quatro dias $(2 \times 31)$. A destilação do solvente sob pressão reduzida forneceu $13 \mathrm{~g}$ de extrato. $9 \mathrm{~g}$ deste extrato foram adsorvidas em $25 \mathrm{~g}$ de sílica gel, a mistura obtida foi colocada sobre papel de filtro num funil de Buchner adaptado num kitazato e sob pressão reduzida eluiu-se com hexano, clorofórmio, acetato de etila e metanol. O material obtido da solução clorofórmica ( $2 \mathrm{~g}$ ) forneceu a mistura das duas isobutilamidas 5 e $\mathbf{6}$ após ser submetido a cromatografia em coluna de sílica gel, usando-se como eluentes misturas de hexano e clorofórmio em polaridade crescente.

\section{AGRADECIMENTOS}

Os autores agradecem ao CNPq, BASA, FUNCAP e FAPERJ pelas bolsas e apoios financeiros concedidos, ao PADETEC (Parque de Desenvolvimento Tecnológico do Ceará) pela obtenção dos GC/ MS, ao CENAUREMN (Centro Nordestino de Aplicação e Uso da ressonância magnética Nuclear) pelos espectros de RMN 1D e 2D e ao Dr. E. F. Guimarães, Museu Nacional do Rio de Janeiro, Brasil, pela identificação da planta.

\section{REFERÊNCIAS}

1. Van Der Berg, M. E.; Silva, M. H. L.; Plantas Medicinais da Amazônia, Belém, CNPq, PTU, Brasil, 1982.

2. Breitmaier, E.; Voelter, W.; Carbon-13 NMR Spectroscopy: High Resolution Methods and Applications in Organic Chemistry and Biochemistry, 3th ed., VCH: Weinhein, 1987.

3. Costa, S. S.; Mors, W. B.; Phytochemistry 1981, $20,1305$.

4. Alencar J. W.; Craveiro, A. A.; Matos F. J. A.; J. Nat. Prod. 1984, 47, 890.

5. Adams R. P.; Identification of Essential Oils by Ion Trap Mass Spectroscopy, Academic Press: London, 1989.

6. Stennhagen, S.; Abrahamson, E.; McLafferty, F. W.; Registry of Mass Spectral Data, J. Willey \& Sons: New York, 1974.

7. Machado, S. M. F.; Militão, J. S. L.T.; Facundo, V. A.; Ribeiro, A.; Morais, S. M.; J. Essent. Oil Res._1994, 6, 643.

8. Agrawal, P. K.; Carbon -13 NMR of Flavonoids, Elsevier: New York, 1989.

9. Pouchert, C. J.; Behnke, J.; The Aldrich Library of ${ }^{13} \mathrm{C}$ and ${ }^{1} \mathrm{H}$ FT NMR Spectra, Aldrich Chemical Company: USA, 1993, vol. 2, 1058B.

10. Parmar, V. S.; Jain, S. C.; Bisht, K. S.; Jain, R.; Taneja, P.; Jha, A.; Tyagi, O.D.; Prasad, A. K.; Wengel, J.; Olsen, C. E; Boll, P. M.; Phytochemistry 1997, 46, 597.

11. Buckingham, J.; Macdonald, F. M.; Bradley, H. M., eds.; Dictionay of Natural Products, Chapman \& Hall: London, 1994, vol. 4, p. 4533; vol. 5 , p. 5561

12. McFerren, M. A.; Rodriguez, E.; J. Ethnopharmacoly 1998, 60, 183.

13. Stöhr, D.; J. D.; Xiao, P. G.; Bauer, R.; J. Ethnopharmacoly 2001, 75, 133. 